\title{
PHARMACOTHERAPY
}

\section{Elevating HDL-cholesterol levels with dalcetrapib is safe}

Statins effectively lower LDL-cholesterol levels, but residual cardiovascular risk remains, particularly in patients with low levels of HDL cholesterol. Inhibition of the cholesteryl ester transfer protein (CETP) with torcetrapib successfully elevated HDL-cholesterol levels, but unexpectedly increased cardiovascular morbidity and mortality. Dalcetrapib is a novel CETP inhibitor, and results from the phase IIb dal-VESSEL trial now show that the drug is safe and tolerable.

Investigators randomly allocated 476 patients with, or at risk of, coronary heart disease, HDL-cholesterol levels $<50 \mathrm{mg} / \mathrm{dl}$, and target LDL-cholesterol levels $(<100 \mathrm{mg} / \mathrm{dl})$ to receive either dalcetrapib $600 \mathrm{mg}$ or placebo daily, in addition to standard therapy, which included statins. After 36 weeks of follow-up, placebocorrected HDL-cholesterol levels had increased by $31 \%(P<0.0001)$.

The placebo-corrected ambulatory systolic and diastolic blood pressures were not statistically different from baseline at 4 weeks (one of the two prespecified end points) or at 36 weeks. Placebocorrected flow-mediated dilatation of the right brachial artery after $5 \mathrm{~min}$ of cuff occlusion-a measure of endothelial function-was also unchanged from baseline at 12 weeks (the other prespecified end point) and at 36 weeks. Additionally, biomarkers of inflammation, oxidative stress, and coagulation were shown to be unchanged at 36 weeks.

These data indicate that dalcetrapib safely increases HDL-cholesterol levels without adversely affecting endothelial function. The long-term efficacy of the drug is being assessed in the phase III dal-PLAQUE 2 and dal-OUTCOMES trials.

Gregory B. Lim

Original article Lüscher, T. F. et al. Vascular effects and safety of dalcetrapib in patients with or at risk of coronary heart disease: the dal-VESSEL randomized clinical trial. Eur. Heart J. doi:10.1093/eurheartj/ehs019 